\title{
Dirección por valores y responsabilidad social en universidades estatales chilenas
}

\author{
Ricardo Gaete Quezada* \\ Universidad de Antofagasta, Antofagasta, Chile. \\ https://orcid.org/0000-0003-2359-2304
}

Recibido: 19/09/2019 Revisado: 11/11/2019 Aceptado: 19/05/2020

\begin{abstract}
Resumen
El objetivo de este artículo es examinar los valores organizacionales, desde la perspectiva de la Dirección por Valores y la Responsabilidad Social Universitaria, declarados por 18 universidades estatales chilenas. Se utiliza un diseño de investigación mixto, mediante un análisis de contenido de planes estratégicos y páginas web institucionales. Los resultados muestran una mayor frecuencia del pluralismo, la democracia y la equidad, mientras que respecto del comportamiento socialmente responsable se destacan la integridad, la excelencia y la libertad. El estudio concluye que las universidades analizadas poseen una identidad corporativa explícita e inteligible, que no incluye mayormente valores y principios de la responsabilidad social universitaria.
\end{abstract}

Palabras clave: ética, valores organizacionales, identidad corporativa, educación superior, universidades estatales.

\section{Management by values and social responsibility in chilean state universities}

\begin{abstract}
The objective of this paper is to describe the organizational values of 18 state universities in Chile from the perspective of Management by Values and Social Responsibility of Universities. This study was conducted using a mixed research design based on the analysis of the contents of universities' strategic plans and webpages. Results show a higher prevalence of pluralism, democracy and equity, whereas socially responsible behavior is characterized by integrity, excellence and freedom. This study concludes that the universities analyzed have an explicit and intelligible corporate identity, even though the latter does not include values and principles of university social responsibility in most cases.
\end{abstract}

Keywords: Ethics, Organizational Values, Corporate Identity, Higher Education, State Universities.

\section{Direção por valores e responsabilidade social em universidades estatais chilenas}

\section{Resumo}

O objetivo do artigo é examinar os valores organizacionais declarados por 18 universidades estatais chilenas, sob a perspectiva da Direção por Valores e a Responsabilidade Social Universitária. Utiliza-se um modelo de pesquisamista, através de uma análise de conteúdo de planos estratégicos e páginas web institucionais. Os resultados mostram uma frequência do pluralismo, a democracia e a equidade, enquanto que com relação ao comportamento socialmente 
responsável destacam a integridade, a excelência e a liberdade. A pesquisa conclui que as universidades analisadas possuem uma identidade corporativa explícita e inteligível que não inclui, em sua maioria, valores e princípios de responsabilidade social universitária.

Palavras-chave: Ética, Valores Organizacionais, Identidade Corporativa, Educação Superior, Universidades Estatais

Como citar:

Gaete-Quezada, R. (2020). Dirección por valores y responsabilidad social en universidades estatales chilenas. Revista Digital de Investigación en Docencia Universitaria, 14(1), e1073. https://doi.org/10.19083/ridu.2020.1073

E. l estudio de la ética del comportamiento de los actores de una comunidad universitaria, es decir, la conducta de los estudiantes, profesores, científicos y la propia universidad, es un ámbito relevante para las Ciencias Sociales, tanto desde la perspectiva del ejercicio profesional como respecto de la cultura y la gestión institucional (Hirsch, 2012).

La perspectiva organizacional del comportamiento ético de las universidades (Gaete, 2011; Vidal-Martínez, 2019), se relaciona con el concepto de Responsabilidad Social Universitaria (RSU), entendida esta última como un compromiso permanente del quehacer universitario con las necesidades y problemáticas de la sociedad, a través de una relación transparente, fluida y constante con el tejido social y sus partes interesadas.

Además, Hirsch (2003) señala que, si bien las instituciones de educación superior (IES) tienen la responsabilidad de proporcionar a la sociedad personas profesionalmente preparadas y con criterio para desarrollar su ejercicio profesional de manera eficiente, también es necesario fortalecer un compromiso social.

Por otra parte, la Dirección por Valores (DpV) y la Identidad Corporativa (IC) son temáticas relevantes para el comportamiento socialmente responsable de las universidades, porque la dimensión ética de la RSU se relaciona con la gestión de los impactos del quehacer universitario en la sociedad (Gaete, 2011, 2016; Jiménez, 2008; Montañez-García, 2017; Pérez-Mato \& Borrazás-González, 2012; Takaki, Bravo, \& Martínez, 2015; Vallaeys, 2008, 2014).

Así, la DpV se presenta como un enfoque de gestión organizacional desarrollado desde fina- les de la década de los noventa, planteado simultáneamente por Dolan y García en España y por Blanchard y O'Connor en EE.UU. (Durán, 2008; García, 2011; Toniut et al., 2015). Otro ejemplo de perspectivas teóricas relevantes en este ámbito, es la propuesta de Rokeach sobre los valores compartidos (Pérez-Mato \& Borrazás-González, 2012).

Montañez-García (2017), resala la importancia de los valores institucionales, debido a que son capaces de contribuir a una mejora en el comportamiento de los individuos en el desempeño de sus funciones y tareas en cada puesto de trabajo. Estos aspectos son determinantes para la realización de las actividades organizacionales.

Además, la $\mathrm{DpV}$ se relaciona con la ética organizacional, especialmente en cuanto a su esencia filosófica y comportamental (Gómez et al., 2018), debido a que los valores organizacionales “...definen el carácter fundamental de cada organización, crean y le dan sentido de identidad a sus miembros, fijan los lineamientos para implementar las prácticas, las políticas y los procedimientos en la actuación cotidiana" (Durán, 2008, p. 356).

Así, los valores organizacionales se transforman en elementos relevantes para la IC (Gaete, 2016; Takaki et al., 2015) y la cultura organizacional de toda institución (Durán, 2008; Llanos, 2018). Según Blanchard y O'Connor (1997), la DpV de una organización considera tres grandes fases:

1. Aclarar propósitos, valores y misión de la organización: decidir y priorizar los valores impulsados en la estrategia organizacional por cada una de sus partes interesadas, así como revisar si la declaración de misión refleja adecuadamente los valores organizacionales.

2. Comunicar la misión y valores organizacio- 
nales: difundir la declaración de misión y valores organizacionales entre las partes interesadas, recopilando los aportes de todos los miembros de la organización para operacionalizar dichas declaraciones en las acciones cotidianas en cada puesto de trabajo.

3. Alinear prácticas organizacionales con la misión y los valores: reducir las brechas entre la misión y valores organizacionales, respecto de las prácticas y conductas individuales, grupales y organizacionales, compatibilizándolos con los propósitos, valores y misión.

Asimismo, Dolan y Altman (2012) afirman que las organizaciones deben considerar cuatro ejes o familias de valores en su DpV:

- Ético-social: modo en el que la gente se comporta y relaciona en un entorno de carácter social o laboral, considerando: honestidad, integridad, respeto, responsabilidad, transparencia o lealtad.

- Económico: aseguran la supervivencia organizacional en un entorno competitivo, guiando las actividades, procesos y resultados esperados, incluye: eficiencia, efectividad, calidad, excelencia, rentabilidad.

- Desarrollo Emocional: relacionado con la motivación intrínseca de las personas, fomentando la iniciativa, innovación y compromiso organizacional, asociado con el optimismo, pasión, autocontrol, paciencia, libertad, felicidad, autonomía, apoyo, iniciativa.

- Espiritual: Capaces de alinear a los otros valores instrumentales, relacionándose con aspectos tales como: respeto por los demás, demostrar un trato justo, expresar interés y preocupación, escuchar con atención, reconocer las contribuciones de los demás y participar en la práctica reflexiva.

Según Dolan y Altman (2012), los valores organizacionales clasificados en los ejes ético-social, económico y desarrollo emocional, poseen un carácter instrumental para las organizaciones laborales, mientras que los valores espirituales poseen la capacidad basal para orientar a los valores instrumentales de cada institución, es decir: “...los valores terminales [espirituales] es lo que se desea, y los instrumentales, la forma en que se consigue lo que se desea" (Durán, 2008, p. 358).

Por otra parte, el interés académico en la IC es creciente en los últimos años, por su relevancia estratégica (Melewar, Karaosmanoglu y Paterson, 2005). En ese sentido, Takaki et al. (2015) señalan que la gestión de la IC se relaciona con cuatro grandes dimensiones: identidad visual, comunicaciones, cultura y comportamiento. Asimismo, la literatura reconoce a los valores organizacionales como un aspecto propio de la cultura corporativa, plasmados en la misión institucional (Durán, 2008; Llanos, 2018; Takaki et al., 2015).

Además, Currás (2010) afirma que la IC es uno de los elementos principales del marketing corporativo. Esto se sostiene en que existe cierta ambigüedad e imprecisión sobre el significado de dicho concepto en la literatura (Melewar et al., 2005). De esta manera, la IC se relaciona fundamentalmente con el ethos institucional, expresado en los valores y la cultura de cada organización, otorgando un carácter distintivo a cada entidad.

Según Melewar et al. (2005), los componentes de la IC son: comunicación corporativa, diseño corporativo, cultura organizacional, comportamiento organizacional, estructura corporativa, identidad industrial y estrategia corporativa. Los mismos autores señalan que los valores y principios son aspectos de la cultura corporativa, afirmando que son fundamentales para desplegar la misión en todos los niveles jerárquicos de la organización.

Asimismo, el concepto de RSU ha alcanzado una madurez epistemológica luego de más de dos décadas de desarrollo a nivel mundial (Baca-Neglia, Rondán-Cataluña \& García del Junco, 2017; Gaete \& Álvarez, 2019; Gómez, Alvarado, \& Pujols, 2018; Martí-Noguera, Calderón, \& Fernández-Godenzi, 2018; Martí-Noguera \& Gaete, 2019; Martí-Noguera \& Martí-Vilar, 2013; Ruiz-Corbella \& Bautista-Cerro, 2016; Vallaeys \& Álvarez, 2019).

Gaete (2011), afirma que los modelos y definiciones de RSU pueden clasificarse en gerenciales, normativas y transformadoras. Esto, con el objetivo de diferenciar las perspectivas que ponen mayor énfasis en la rendición de cuentas sobre los impactos organizacionales, los aspectos deontológicos y el desarrollo sostenible respectivamente (Gaete \& Álvarez, 2019; Vallaeys \& Álvarez, 2019).

Actualmente, algunas concepciones sobre la RSU reconocen a la educación superior como un 
bien común (Licandro \& Yepes, 2018; Martí-Noguera, Licandro, \& Gaete-Quezada, 2018), en contraste con aquellas posturas científicas que critican la excesiva mercantilización de las universidades a nivel mundial (Valleys, 2014).

Desde una perspectiva epistemológica, algunas líneas de investigación de la RSU relacionadas con su dimensión ética-normativa son: 1) influencia de los valores en el comportamiento socialmente responsable en los estudiantes (Arango et al., 2014; Martí-Noguera, Martí-Vilar, \& Almerich, 2014; Navarro et al., 2010, 2012); 2) ética profesional en la formación universitaria (Hirsch, 2012; Hirsch \& Pérez-Castro, 2019; Martí-Vilar \& Rodríguez-Osorio, 2016) y 3) gestión estratégica de las universidades (Gaete, 2011, 2015; Mendoza-Fernández et al., 2015; Valarezo \& Núñez, 2014; Vallaeys, 2008, 2018).

En el contexto anterior, una de las definiciones clásicas de la RSU planteada por Vallaeys (2008), es considerada como una nueva filosofía de gestión ética e inteligente para las universidades:

Una política de mejora continua de la universidad hacia el cumplimiento efectivo de su misión social mediante 4 procesos: Gestión ética y ambiental de la institución; Formación de ciudadanos responsables y solidarios; Producción y difusión de conocimientos socialmente pertinentes; Participación social en promoción de un Desarrollo más humano y sostenible. (p. 209)

De acuerdo con Martínez-Usarralde, Lloret-Catalá y Mas-Gil (2017), la RSU desde una perspectiva integradora implica “...una política institucional integral que se encarga de la gestión de todos los impactos sociales que la universidad genera, en diálogo participativo con los diversos grupos de interés" (p. 5), relacionando el comportamiento socialmente responsable con los procesos de toma de decisiones que afectan a las partes interesadas en el quehacer universitario.

Según Gaete (2016), la RSU encuentra sus orígenes en América Latina en el proyecto Universidad Construye País (UCP), desarrollado en Chile entre los años 2001 al 2008. Dicha iniciativa propone un conjunto de valores y principios (ver Figura 1) que orientan el comportamiento socialmente responsable de las universidades y los miembros de sus respectivas comunidades universitarias (Jiménez, 2008).

El proyecto UCP contribuye al fortalecimiento de la ética en las universidades, proponiendo una perspectiva normativa de la RSU (Gaete, 2011), entendida como:

La capacidad que tiene la universidad como institución, de difundir y poner en práctica un conjunto de principios y valores generales y específicos por medio de los procesos clave de gestión, docencia, investigación y extensión, respondiendo socialmente así ante la comunidad universitaria y el país en que está inserta (Fernández, Delpiano \& De Ferrari, 2006, p.14).

Sin embargo, Vallaeys (2018) afirma que el compromiso ético de la universidad es una de las diez

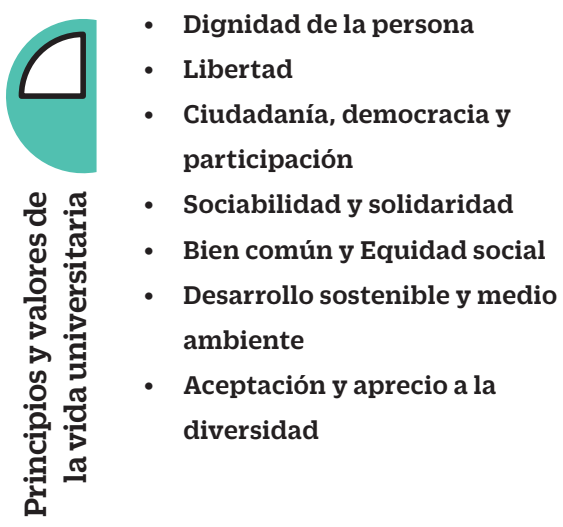

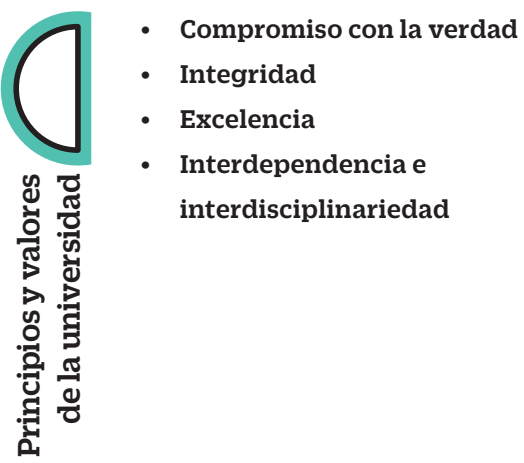

Figura 1. Principios y valores Proyecto Universidad Construye País, elaboración propia, basado en Jiménez (2008). 
falacias del significado de la RSU, postulando que “... hay una relación intrínseca entre ética de la responsabilidad, definición de la responsabilidad social en términos de responsabilidad por los impactos y deber de vigilancia y asociación inter-organizacional para resolver los problemas de justicia y sostenibilidad del conjunto social" (p. 40), destacando que el comportamiento socialmente responsable de las universidades debe orientarse hacia el desarrollo más sostenible de la sociedad, como un imperativo ético del quehacer universitario.

De esta manera, nuestro estudio intenta responder a las siguientes interrogantes: ¿Cuáles son los principales valores organizacionales que orientan el quehacer de las universidades estatales chilenas (UECH)?, ¿Cuáles son los valores y principios de la DpV y la RSU que las UECH incorporan en su identidad corporativa?

\section{Diseño}

Se desarrolla un diseño de investigación mixto, combinando las miradas cuantitativas y cualitativas en la etapa empírica del estudio (Núñez, 2017; Piñuel, 2002), para examinar los valores organizacionales de las UECH en su IC declarada en planes estratégicos o páginas web institucionales.

La población objeto de estudio son las universidades chilenas, que según el Ministerio de Educación son 57 instituciones reconocidas para el año 2019. Los criterios de inclusión en la muestra son: 1) ser una universidad estatal y 2) tener disponible en sus páginas web institucionales los planes estratégicos o la declaración de sus valores organizacionales. Según la Tabla 1, la muestra del estudio está compuesta por 18 universidades estatales

Tabla 1

Universidades estatales chilenas incluidas en el estudio.

\begin{tabular}{|c|c|c|c|}
\hline Universidad & $\begin{array}{l}\text { Año } \\
\text { creación }\end{array}$ & $\begin{array}{l}\text { Acreditación } \\
\text { institucional }\end{array}$ & Documento consultado \\
\hline Arturo Prat & 1984 & 4 años (2017-2021) & Plan estratégico 2014-2020 \\
\hline de Tarapacá & 1981 & 5 años (2017-2022) & Plan estratégico 2017-2022 \\
\hline de Antofagasta & 1981 & 5 años (2017-2022) & Plan estratégico 2016-2020 \\
\hline de Atacama & 1981 & 3 años (2017-2020) & Plan estratégico 2015-2019 \\
\hline de La Serena & 1981 & 4 años (2016-2020) & Plan estratégico 2016-2020 \\
\hline de Chile & 1842 & 7 años (2018-2025) & Plan estratégico 2017-2026 \\
\hline de Santiago & 1947 & 6 años (2014-2020) & Plan estratégico 2016-2020 \\
\hline $\begin{array}{l}\text { Metropolitana de Ciencias de la } \\
\text { Educación (UMCE) }\end{array}$ & 1985 & 3 años (2016-2019) & Plan estratégico 2016-2020 \\
\hline $\begin{array}{l}\text { Tecnológica Metropolitana } \\
\text { (UTEM) }\end{array}$ & 1993 & 4 años (2016-2020) & Plan estratégico 2016-2020 \\
\hline de Valparaíso & 1981 & 5 años (2017-2022) & Página web institucional \\
\hline de Playa Ancha & 1985 & 5 años (2016-2021) & Plan estratégico 2016-2025 \\
\hline de O’Higgins & 2015 & Sin acreditación & Plan estratégico 2017-2019 \\
\hline de Talca & 1981 & 6 años (2019-2025) & Plan estratégico 2016-2020 \\
\hline del Biobío & 1981 & 5 años (2019-2024) & Plan estratégico 2015-2019 \\
\hline de la Frontera & 1981 & 6 años (2018-2024) & Plan estratégico 2013-2023 \\
\hline de los Lagos & 1993 & 4 años (2016-2020) & Plan estratégico 2013-2018 \\
\hline de Magallanes & 1981 & 4 años (2015-2019) & Plan estratégico 2017-2017 \\
\hline de Aysén & 2015 & Sin acreditación & Plan estratégico 2019-2023 \\
\hline
\end{tabular}

Fuente: elaboración propia. 


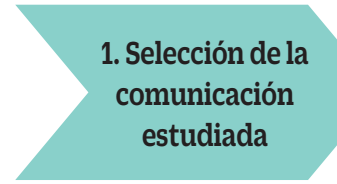

- Planes estratégicos y sitios web de las UECH, consultados hasta el día 30 de octubre de 2019.

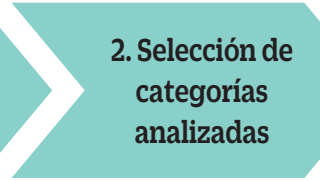

- Dirección por valores y responsabilidad social universitaria, como modelos teóricos relacionados con la IC de las UECH.

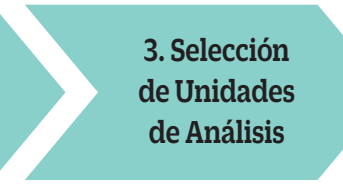

•Valores y
principios
institucionales
declarados por las
UECH en sus planes
estratégicos o
páginas web.

- Análisis cuantitativo, mediante procedimientos de estadística descriptiva, para identificar frecuencia de los valores organizacionales.

Figura 2. Procedimiento aplicación del análisis de contenido, elaboración propia, basado en Piñuel (2002).

(Gaete, 2015; Gil, 2012; Mendoza-Fernández et al., 2015; Núñez, Alonso, \& Pontones, 2015).

La técnica de recolección de la información del estudio es el análisis de contenido, que según $\mathrm{Pi}$ ñuel (2002) considera cuatro pasos fundamentales en el procedimiento de aplicación de dicha técnica:

Así, mediante el procedimiento descrito en la Figura 2, se analizan los planes estratégicos y páginas web de las UECH incluidas en el estudio (ver Tabla 1), según las tipologías de valores y principios de Dolan y Altman (2012) y de Jiménez (2008) respectivamente, apoyado con la elaboración de tablas y figuras a través del software MS EXCEL, además de la triangulación con los conceptos y modelos teóricos expuestos en la introducción.

\section{Resultados}

La Tabla 2, presenta la distribución de los valores organizacionales declarados por las instituciones de la muestra, analizando la distribución porcentual de los valores organizacionales según la propuesta de ejes elaborada por Dolan y Altman (2012).

Según la Tabla 2, la Universidad del Biobío es la institución con la mayor cantidad de valores organizacionales, observándose una media de 7,4 valores por cada UECH incluidas en el estudio. En cuanto a los ejes valóricos, todas las UECH declaran al menos un valor de carácter espiritual como expresión de la IC de este tipo de IES (Blanchard
\& O’Connor, 1997; Fernández, 2010; Morales et al., 2017; Tataki et al., 2015).

Asimismo, los valores organizacionales se distribuyen mayoritariamente entre los ejes ético-social $(37,6 \%)$ y espiritual (38,3\%), donde el $33 \%$ de las UECH declaran valores organizacionales en los cuatro ejes del modelo de Dolan y Altman (2012).

La Figura 3, presenta la distribución de los valores con mayor frecuencia en la IC de las UECH, destacándose el Pluralismo presente en el 72\% de los planes estratégicos institucionales y páginas web analizadas, como principal valor organizacional de tipo espiritual, según la clasificación de Dolan y Altman (2012).

Así, este resultado es concordante con lo planteado por Solari (2016) en cuanto a la importancia del pluralismo intelectual y la libertad académica con las que deben caracterizarse y funcionar las IES en un sistema universitario como el chileno. Lo anterior, también es ratificado por el art. $5^{\circ}$ de la Ley $\mathrm{N}^{\circ} 21.094$ sobre universidades estatales del Ministerio de Educación del año 2018, que reconoce al Pluralismo como uno de los valores y principios distintivos de las UECH.

Además, algunas ideas sobre el significado del Pluralismo presente en las declaraciones de los planes estratégicos y páginas web analizados, lo relacionan con el respeto por la diversidad en sus distintas formas de expresión:

Reconoce y acoge, con respeto y tolerancia, la existencia de diferentes pensamientos e ideologías en su seno, valora y protege la 
Tabla 2

Valores universidades estatales según ejes de la Dirección por valores.

\begin{tabular}{|c|c|c|c|c|c|}
\hline \multirow[b]{2}{*}{ Universidad } & \multirow{2}{*}{$\begin{array}{c}\text { Cantidad } \\
\text { de Valores } \\
\text { declarados }\end{array}$} & \multicolumn{4}{|c|}{ Distribución \% según ejes valóricos } \\
\hline & & Ético-social & Económico & $\begin{array}{l}\text { Desarrollo } \\
\text { emocional }\end{array}$ & Espiritual \\
\hline Arturo Prat & 7 & $57 \%$ & 0 & $12 \%$ & $29 \%$ \\
\hline de Tarapacá & 5 & 0 & $40 \%$ & 0 & $60 \%$ \\
\hline de Antofagasta & 6 & $50 \%$ & $17 \%$ & 0 & $33 \%$ \\
\hline de Atacama & 6 & $50 \%$ & 0 & $17 \%$ & $33 \%$ \\
\hline de La Serena & 4 & $50 \%$ & 0 & $25 \%$ & $25 \%$ \\
\hline de Chile & 9 & $44,4 \%$ & 22,2 & 0 & $33,3 \%$ \\
\hline de Santiago & 8 & $50 \%$ & $12,5 \%$ & $12, \%$ & $25 \%$ \\
\hline UMCE & 5 & $40 \%$ & 0 & $20 \%$ & $40 \%$ \\
\hline UTEM & 6 & $43 \%$ & $14 \%$ & 0 & $43 \%$ \\
\hline de Valparaíso & 9 & $33,3 \%$ & 0 & $22,2 \%$ & $44,4 \%$ \\
\hline de Playa Ancha & 8 & $37,5 \%$ & $12,5 \%$ & 0 & $50 \%$ \\
\hline de O'Higgins & 7 & $28,6 \%$ & $14 \%$ & $28,6 \%$ & $28,6 \%$ \\
\hline de Talca & 8 & $37,5 \%$ & 12,5 & $25 \%$ & $25 \%$ \\
\hline del Biobío & 12 & $50 \%$ & $16,6 \%$ & $16,6 \%$ & $16,6 \%$ \\
\hline de la Frontera & 11 & $36 \%$ & $9 \%$ & $9 \%$ & $46 \%$ \\
\hline de los Lagos & 5 & $40 \%$ & $20 \%$ & 0 & $40 \%$ \\
\hline de Magallanes & 4 & 0 & 0 & $25 \%$ & $75 \%$ \\
\hline de Aysén & 7 & $29 \%$ & $14 \%$ & $14 \%$ & $43 \%$ \\
\hline
\end{tabular}

Fuente: elaboración propia.

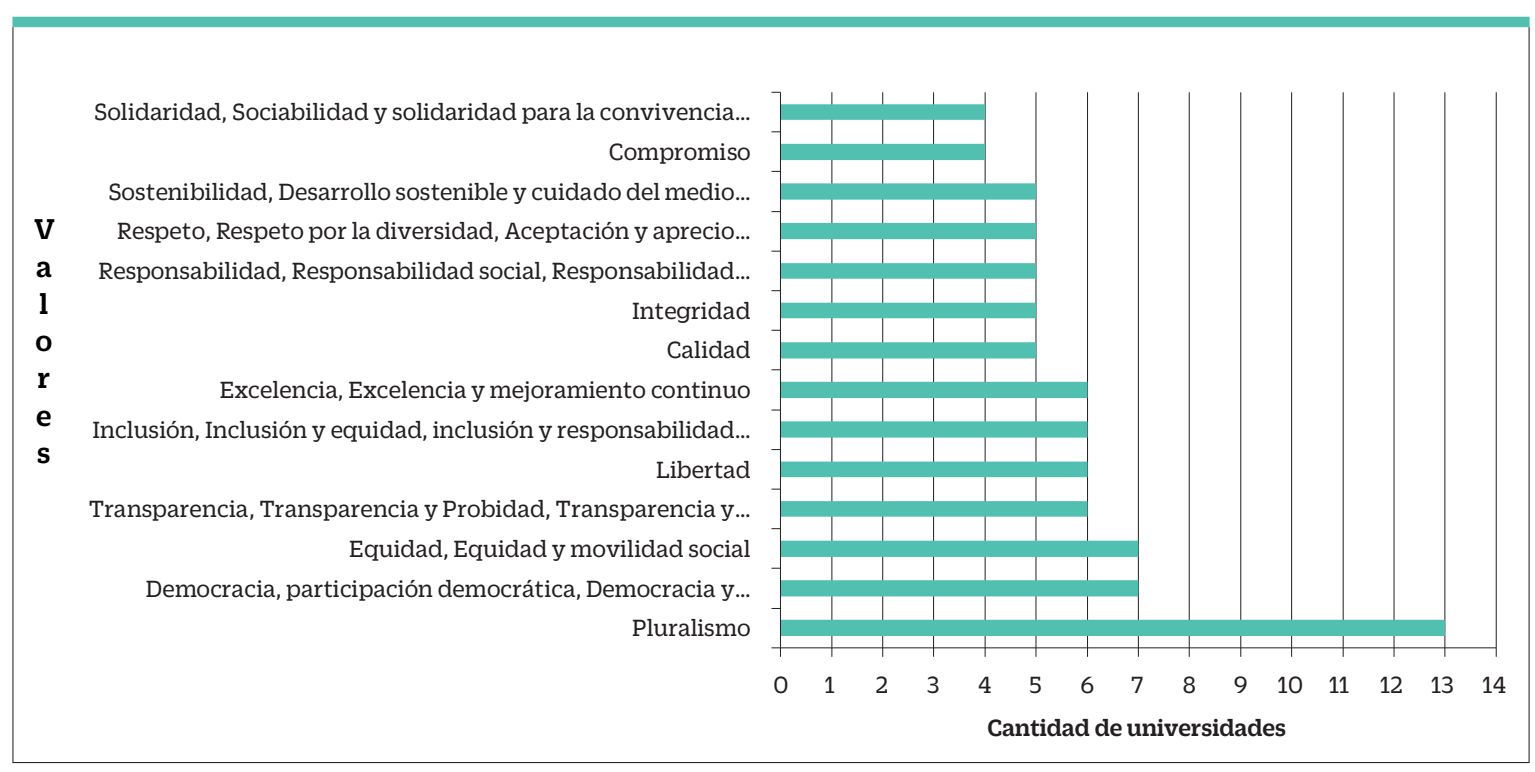

Figura 3. Distribución de universidades estatales chilenas según valores institucionales, elaboración propia. 
heterogeneidad nacional, social, cultural, religiosa, de género y étnica de sus miembros, y los derechos que les son propios (Universidad de Antofagasta)

Implica aceptar las diferentes ideas, credos y posiciones de distinta naturaleza en un marco de tolerancia mutua con el propósito de generar conocimientos, aprendizajes y saberes a través del diálogo y del intercambio académico (Universidad de Valparaíso)

También, la Figura 3 muestra que el 39\% de las UECH incluyen a la Equidad como uno de los valores organizacionales de su IC, identificándose los siguientes ejemplos de definiciones en los planes estratégicos y páginas web:

Acceso a la formación de diversos grupos, generando las condiciones y entregando los soportes institucionales para estos fines, como, asimismo, en las condiciones internas de la organización (Universidad Arturo Prat)

Acceso igualitario a postulantes provenientes de distintos estratos socioeconómicos, lograr que permanezcan en su carrera hasta el término de sus estudios, que tengan un aprendizaje similar, independiente de su trayectoria de vida (Universidad de Valparaíso)

La Equidad, como un valor organizacional, establece un desafío importante para las UECH desde una perspectiva ética de su comportamiento socialmente responsable, en lo relativo al acceso a la educación superior en Chile (Beltrán-Llevador, Íñigo-Bajo \& Mata-Segreda, 2014; Espinoza, 2017; Espinoza \& González, 2016).

Según el Ministerio de Educación (2019), desde la perspectiva de la equidad en el acceso a la educación superior chilena, solo el 33\% de los estudiantes que ingresaron a las UECH el año 2018 provienen de establecimientos educacionales de carácter estatal, que forman a las personas más vulnerables y de escasos recursos en Chile, mientras que el $64,7 \%$ provienen de establecimientos particulares o particulares subvencionados.

Asimismo, el Ministerio de Educación (2019) señala que dentro de las UECH que declaran a la equidad como un valor, la Universidad de Valpa- raíso solo registra un $19,7 \%$ de estudiantes provenientes de establecimientos estatales, mientras que la Universidad de Chile es la que registra el mayor porcentaje de estudiantes que provienen de establecimientos privados, con un 32,6\%.

También, en la Figura 3 se evidencia que el 39\% de las universidades de la muestra incluyen a la Democracia como uno de los valores organizacionales de su IC, la que es definida por algunas universidades como:

Su organización se basa en la convivencia y el ejercicio democrático de elección de sus gobernantes y autoridades (Universidad de Aysén)

Valora y promueve que todas las visiones formen parte de los procesos de la toma de decisiones del gobierno universitario (Universidad de Los Lagos)

En virtud de lo anterior, el gobierno universitario y las personas que gestionan a las UECH, se transforman en un indicador importante para la democracia universitaria. En ese sentido, la distribución por género de las autoridades unipersonales tales como Rector o Decano, se encuentra compuesto mayoritariamente por hombres en la actualidad (Gaete, 2015), contraviniendo el necesario pluralismo y equidad con las que dichas instancias de alta dirección debieran tener al interior de las UECH.

La Universidad de Aysén es la única UECH cuya máxima autoridad unipersonal es una mujer, considerándose una instancia de equidad de género que debe replicarse por una mayor cantidad universidades, debido a que el rol de los directivos y en general de las instancias de gobierno corporativo, se transforman en un aspecto relevante tanto para la DpV como para la RSU (Baca-Neglia et al., 2017; Blanchard \& O'Connor, 1997; Dolan \& Altman, 2012; Gaete, 2015; Núñez, Alonso, \& Pontones, 2015).

Por ello, la existencia de valores organizacionales de carácter espiritual según el modelo de Dolan y Altman (2012), permiten a las UECH orientar su IC hacia el pluralismo, la democracia o la equidad como elementos culturales esenciales, equilibrando el impacto de otros valores más instrumentales como la eficiencia o la calidad, habitualmente relacionados con una mayor mercantilización de la educación superior (Bel- 
trán-Llevador et al., 2014; Vallaeys, 2014).

Respecto de la RSU, la Figura 4 expone los resultados obtenidos en nuestro estudio sobre los valores y principios del proyecto UCP, destacando las mayores frecuencias de la Libertad, Excelencia e Integridad. Los resultados de la Figura 4, plantean un desafío importante para la gestión del cambio de la IC de las UECH, que oriente su quehacer institucional hacia la perspectiva ética de la RSU, donde los valores organizacionales adquieren un rol fundamental (Beltrán-Llevador et al., 2014; Gaete, 2016; Jiménez, 2008; Rubio, Améstica-Rivas, King-Domínguez, \& Ganga-Contreras, 2019; Tataki et al., 2015).

Así, algunos estudios como el de Navarro et al., (2012) sobre los valores y la RSU, confirman la relación existente entre valores, actitudes y comportamiento socialmente responsable de los estudiantes universitarios chilenos. Estos destacan a la Libertad como el valor que consideran más importante respecto del modelo propuesto por la UCP, coincidiendo con los resultados de nuestro estudio que señala que la IC del 33\% de las UECH incluye a dicho valor en sus planes estratégicos o páginas web.

Según Fernández, Delpiano y De Ferrari (2006), dicho valor organizacional en el ámbito universitario, se aplica a cuestiones tales como: pensamiento, religión, investigación, opinión, enseñanza, reunión y asociación, entre otras. Asimismo, según los planes estratégicos y páginas web de las instituciones incluidas en nuestro estudio implica:

[Un] espacio abierto para ejercer la función académica, y de límite ético y procedimental de las prácticas llevadas a cabo en el cumplimiento de los objetivos académicos (Universidad de La Serena)

Respetar la diversidad de creencias, posiciones, pensamientos y opiniones de sus integrantes, como también aquellas opciones que tomen frente a la vida y a los procesos formativos, en la medida que estas aporten al logro del bien común (Universidad de Magallanes)

También, la Libertad es un valor importante para las UECH porque se encuentra incluido en el art. $5^{\circ}$ de la Ley $\mathrm{N}^{\circ} 21.094$ sobre universidades estatales, con dos formas de expresión: Libertad de pensamiento y de expresión; Libertad de cátedra (Ministerio de Educación, 2018). Además, la Libertad debiera ser un aspecto distintivo del comportamiento socialmente responsable de las IES, fuertemente arraigada en la IC de las UECH tanto desde la perspectiva legal como en el ámbito de su gestión institucional (Jiménez, 2008; Navarro et al., 2012).

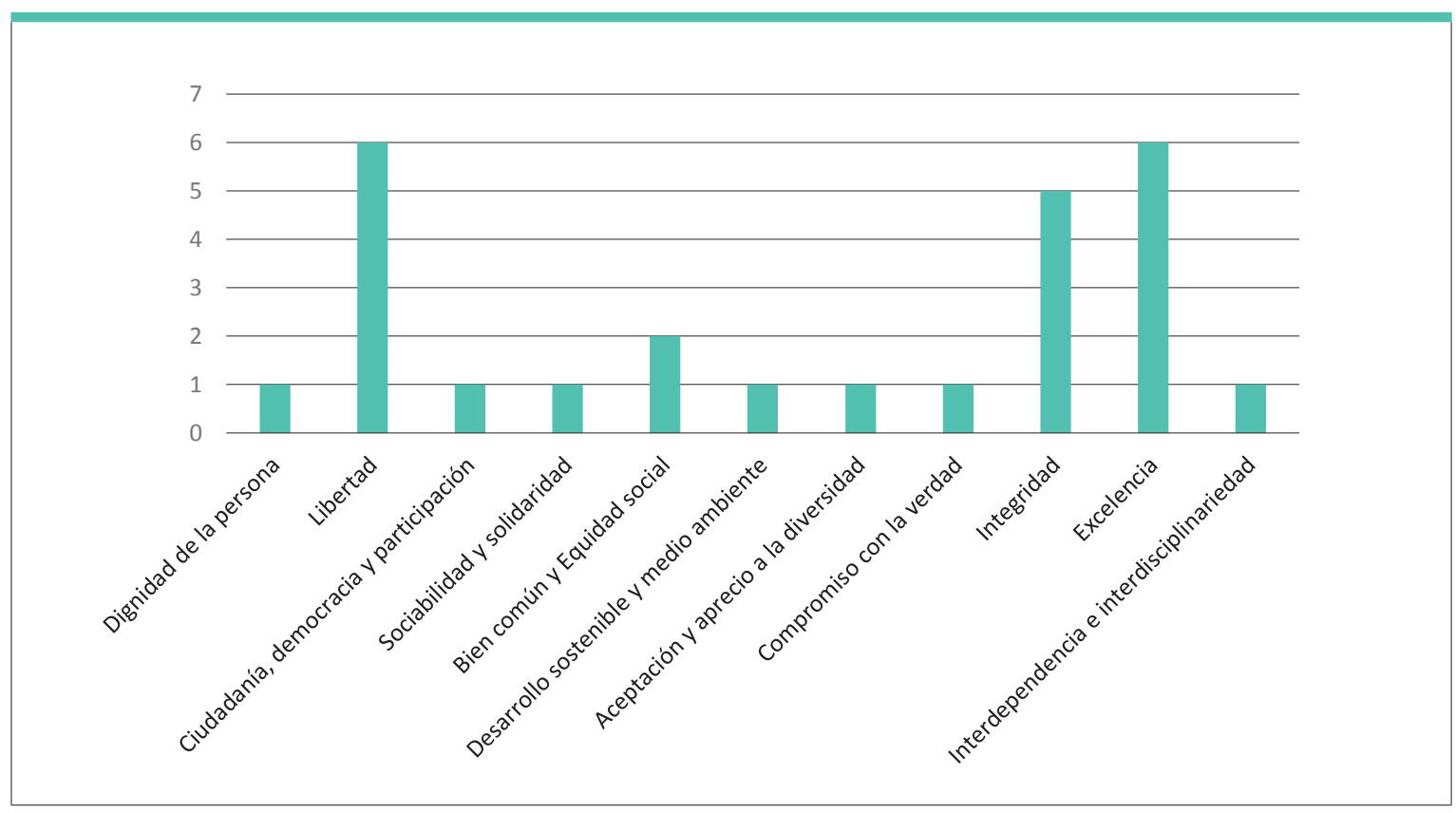

Figura 4. Universidades según valores y principios proyecto UCP, elaboración propia. 
Asimismo, la Excelencia es otro de los valores y principios del proyecto UCP que el 33\% de las UECH incluyen en su IC. Según Fernández, Delpiano y De Ferrari (2006), la Excelencia es entendida como la “...calidad o bondad superior que distingue y hace objeto de especial aprecio al quehacer de personas u organizaciones. Atañe tanto a los fines, medios y procedimientos y se expresa en la gestión y en las funciones universitarias" (p. 56). Algunos ejemplos sobre la definición de la Excelencia en los planes estratégicos y páginas web la describen como:

La inspiración que hace posible la permanente búsqueda de la perfección. Desafía el desempeño de las personas y la Institución en el cumplimiento de su misión y visión. Este valor supone el esfuerzo individual de cada miembro de la comunidad universitaria y del mismo modo, un sentido de conjunto y convergencia en la búsqueda de la excelencia (Universidad de Talca)

Mejoramiento continuo en el desarrollo de su quehacer institucional, propendiendo a alcanzar los mayores niveles en su labor académica (Universidad de Tarapacá)

De esta manera, la excelencia se transforma en un aspecto cultural relevante para la IC de las UECH, que se extiende hacia todas las funciones del quehacer universitario. Según lo establecido por la Ley 21.094 sobre universidades estatales, es a través del aseguramiento de la calidad y los procesos de acreditación tanto institucional como de programas de pre y postgrado, que existe una relación directa entre la dirección estratégica de una universidad y su calidad institucional (Rodríguez-Ponce \& Pedraja-Rejas, 2013), con el objetivo de transformarse en organizaciones más responsables y eficientes (Baca-Neglia et al., 2017; Bonifaz \& Barba, 2019).

Finalmente, según la Figura 4, la Integridad surge como un tercer valor organizacional con mayor frecuencia relacionado con el comportamiento socialmente responsable de las UECH. Según el proyecto UCP, la Integridad se define como “...cualidad de personas e instituciones que son coherentes con sus principios y valores, tanto en sus declaraciones como en sus acciones; siendo rectas, transparentes y honestas" (Fernández, Delpiano \& De Ferrari, 2006, p. 54).
Así, el 28\% de las UECH declaran este aspecto en su IC, donde algunas definiciones contenidas en los planes estratégicos y páginas web señalan que la integridad implica:

La congruencia entre lo que se manifiesta y lo que se hace. Significa actuar con honestidad, hacer lo correcto sin afectar a otras personas. Una persona íntegra tiene como recompensa un desempeño consciente $\mathrm{y}$ de respeto hacia los demás, generando confianza y armonía con y entre sus pares (Universidad de Talca)

Actitud ética transversal, conformada por elementos como la honestidad, la verdad, la probidad (Universidad Metropolitana de Ciencias de la Educación)

Respecto de lo anterior, la transparencia (Pérez, 2017) y la rendición de cuentas (Guijarro, Gomera, \& Antúnez, 2016; Núñez, Alonso, \& Pontones, 2015; Huerta-Riveros, 2017), se transforman en indicadores importantes de la Integridad en el contexto de la RSU (Beltrán-Llevador et al., 2014; Gaete, 2011).

Según el Consejo para la Transparencia (2017), las UECH alcanzan un promedio de cumplimiento del $82,3 \%$ de las normas de transparencia activa estipuladas en la Ley 20.285 sobre Transparencia y Probidad, donde la Universidad de Talca $(96,5 \%)$ posee un alto porcentaje de cumplimiento, mientras que la Universidad del Biobío $(65,88 \%)$ tiene el grado de cumplimiento más bajo dentro de las UECH.

\section{Conclusiones}

El modelo de DpV de Dolan y Altman (2012), establece como una consideración fundamental que la IC debe alcanzar un equilibrio entre los valores espirituales y aquellos relacionados con los ejes ético-social, económico y de desarrollo emocional, posibilitando a las organizaciones un desempeño más ético de su misión y visión institucional.

En ese sentido, nuestro estudio evidencia que las UECH presentan un núcleo de valores espirituales que alinean y orientan su quehacer, agregando un conjunto de valores de carácter instrumental que 
configuran una IC explícita e inteligible, que encuentra en el pluralismo el principal valor organizacional distintivo de las universidades estudiadas.

Sin embargo, un aspecto deficitario de las UECH en el contexto del pluralismo, la democracia y la equidad como principales valores organizacionales instrumentales, que promueven la participación equitativa y plural de hombres y mujeres en los cargos directivos de gobierno universitario. Resultará imprescindible aumentar la cantidad de mujeres en puestos de toma de decisiones de carácter unipersonal, tanto en rectorías como decanaturas, plasmando efectivamente dichos valores en el funcionamiento de cada UECH.

Además, la IC de la mayoría de las UECH no muestra gran coincidencia con los principios y valores de la RSU considerados en el proyecto UCP, lo que queda de manifiesto por ejemplo en su no incorporación en la Ley 21.094 sobre universidades estatales, aprobada recientemente el año 2018.

No obstante, los resultados de nuestro estudio destacan la libertad, la excelencia y la integridad como expresión del comportamiento socialmente responsable de las universidades analizadas, donde la excelencia es un valor organizacional especialmente distintivo de las UECH, por su trascendencia para las políticas públicas de acreditación de calidad y gratuidad de la educación superior implementadas en Chile en la última década.

Por ello, resulta recomendable que las universidades que gestionen su IC a través de la DpV, consideren un conjunto de valores y principios conocidos claramente por todos los miembros de la comunidad universitaria, que sustenten las políticas y procedimientos emanados desde la misión y visión institucional, equilibrando los valores espirituales e instrumentales que orienten el comportamiento socialmente responsable hacia el bien común y el desarrollo sostenible de la sociedad (Beltrán-Llevador et al., 2014; Gaete \& Álvarez, 2019; Licandro \& Yepes, 2018; Vallaeys \& Álvarez, 2019).

Finalmente, las limitaciones de nuestro estudio se vinculan fundamentalmente con las posibilidades de generalización de nuestros resultados mediante inferencias estadísticas. Desde esa perspectiva, es recomendable desarrollar nuevos estudios que incorporen una cantidad estadísticamente representativa de sujetos, mediante el diseño, validación y aplicación de un instrumento que mida el compromiso y adhesión de los miembros de las UECH con los valores y principios declarados en la IC de cada institución.

\section{Referencias}

Arango, O., Clavijo, S., Puerta, I., \& Sánchez, J. (2014). Formación académica, valores, empatía y comportamientos socialmente responsables en estudiantes universitarios. Revista de la Educación Superior, 43(1), 89-105. https://doi.org/10.1016/j.resu.2015.01.003

Baca-Neglia, H., Rondán-Cataluña, F., \& García del Junco, J. (2017). Propuesta de medición de la responsabilidad social universitaria. Revista Espacios, 38(43), 12-49. Recuperado de https://www.revistaespacios. com/a17v38n43/a17v38n43p12.pdf

Blanchard, K. \& O'Connor, M. (1997). Administración por valores. Bogotá: Norma.

Beltrán-Llevador, J., Íñigo-Bajo, E., \& Mata-Segreda, A. (2014). La responsabilidad social universitaria, el reto de su construcción permanente. Revista Iberoamericana de Educación Superior (RIES), 14(6), 3-18. https:// doi.org/10.22201/iisue.20072872e.2014.14.128

Bonifaz, E. \& Barba, E. (2019). La calidad de la educación universitaria: una visión desde el direccionamiento y la gestión estratégica. Revista Boletín REDIPE, 8(3), 106-116. Recuperado de https://revista.redipe.org/ index.php/1/article/view/699

Consejo para la Transparencia (2017). Informes sobre fiscalización del cumplimiento de las normas sobre transparencia activa Ley 20.285. Recuperado de https://www.portaltransparencia.cl/PortalPdT/pdtta/-/ta/CTO01/ARCPLT/AREST/37739836

Currás, R. (2010). Identidad e imagen corporativas: revisión conceptual e interrelación. Revista Teoría y Praxis, 7, 9-34. https://doi.org/10.22403/UQROOMX/ TYP07/01

Dolan, S. \& Altman, Y. (2012). Managing by values: The leadership spirituality connection. People \& Strategy, 35(4), 20-26. Recuperado de https:/go. gale.com/ps/anonymous?id=GALE\%7CA324980929\& sid=googleScholar\&v=2.1\&it=r\&linkaccess=abs\&iss $\mathrm{n}=19464606 \& \mathrm{p}=\mathrm{AONE} \& \mathrm{sw}=\mathrm{w}$

Durán, M. (2008). La administración por valores: una metodología humanista de cambio cultural en la empresa. Revista Ciencias Económicas, 26(2), 355-375. Recuperado de https://revistas.ucr.ac.cr/index.php/ 
economicas/article/view/7149

Espinoza, O. (2017). Acceso al sistema de educación superior en Chile. El tránsito desde un régimen de elite a uno altamente masificado y desregulado. Revista Universidades, 74, 7-30. Recuperado de https://www. redalyc.org/pdf/373/37354774003.pdf

Espinoza, O. \& González, L. (2016). La educación superior en Chile y la compleja transición desde el régimen de autofinanciamiento hacia el régimen de gratuidad. Revista Latinoamericana de Educación Comparada, 7(10), 35-51. Recuperado de https://dialnet.unirioja. es/servlet/articulo?codigo $=6562408$

Fernández, R. (2010). Criterios para la evaluación de planes estratégicos de unidades académicas. Calidad en la Educación, (32), 206-233. https://doi.org/10.31619/ caledu.n32.157

Fernández, C., Delpiano, C., \& De Ferrari, J. (2006). Responsabilidad Social Universitaria Una manera de ser universidad. Teoría y práctica en la experiencia chilena. Recuperado de http://dspace.utalca.cl/bitstream/1950/10293/1/Responsabilidad\%20social\%20 universitaria.pdf

Gaete, R. (2011). La responsabilidad social universitaria como desafío para la gestión estratégica de la Educación Superior: el caso de España. Revista de Educación, 355, 109-133. Recuperado de https://recyt. fecyt.es/index.php/Redu/article/view/10478

Gaete, R. (2015). Responsabilidad social en el gobierno y gestión de las universidades estatales chilenas. Revista Civilizar, 15(29), 163-180. https://doi. org/10.22518/16578953.482

Gaete, R. (2016). La responsabilidad social universitaria en la identidad corporativa de las universidades chilenas. Un análisis de contenido. Revista Documentos y Aportes en Administración Pública y Gestión Estatal, 26, 43-74. https://doi.org/10.14409/ da.v16i26.5935

Gaete, R. \& Álvarez, J. (2019). Responsabilidad social universitaria en Latinoamérica. Los casos de URSULA y AUSJAL. Revista Actualidades Investigativas en Educación, 19(3), 1-27. https://doi.org/10.15517/aie. v19i3.38637

García, S. (2011). Dirección por valores. Barcelona: Universidad de Barcelona. Recuperado de http://www.salvadorgarcia.eu/img/secciones_pdfs/Direccion\%20por\%20 Valores.\%20Salvador\%20Garcia.\%20UB,\%202011.pdf

Gil, I. (2012). El rol de las universidades públicas frente a la responsabilidad social universitaria. Revista Panorama Económico, 20, 235-250. https://doi.or- g/10.32997/2463-0470-vol.20-num.0-2012-346

Gómez, L. M., Alvarado, Y., \& Pujols, A. (2018). Practicando la responsabilidad social universitaria en el Caribe: Perspectivas de los públicos internos. Revista Digital de Investigación en Docencia Universitaria, 12(1), 96-118. https://doi.org/10.19083/ridu.12.714

Gómez, M., Vidal, C., Ureña, Y., \& Acevedo, C. (2018). Administración por valores: Estrategia de mejoramiento del clima organizacional en las empresas. Revista Espacios, 39(18), 23-26. Recuperado de https://www. revistaespacios.com/a18v39n28/a18v39n28p23.pdf

Guijarro, C., Gomera, A., \& Antúnez, M. (2016). Propuesta de indicadores de la responsabilidad social universitaria conforme a la guía G4 del GRI: el caso de la Universidad de Córdoba. CIRIEC-Revista de Economía Pública, Social y Cooperativa, 87, 103-137. Recuperado de https://ojs.uv.es/index.php/ciriecespana/article/view/6861/10876

Hirsch, A. (2003). Elementos significativos de la ética profesional. Reencuentro, 38, 8-15. Recuperado de https://www.redalyc.org/pdf/340/34003802.pdf

Hirsch, A. (2012). Conductas no éticas en el ámbito universitario. Perfiles Educativos, vol.34, número especial, 142-152. Recuperado de http://www.scielo.org.mx/ pdf/peredu/v34nspe/v34nspea13.pdf

Hirsch, A. \& Pérez-Castro, J. (2019). Ética profesional y responsabilidad social universitaria: experiencias institucionales. Instituto de Investigaciones sobre la Universidad y la Educación: Universidad Nacional Autónoma de México.

Huerta-Riveros, P. \& Gaete-Feres, H. (2017). Responsabilidad social universitaria a través de los reportes de sostenibilidad del Global Reporting Initiative. Experiencia de una universidad pública. Revista Iberoamericana de Educación Superior, 23(8), 120-137. https:// doi.org/10.19083/ridu.12.714

Jiménez, M. (2008). ¿Cómo medir la percepción de la responsabilidad social en los diversos estamentos de la universidad?: una experiencia concreta. Revista Educación Superior y Sociedad, 13(2), 139-161. Recuperado de https://unesdoc.unesco.org/ark:/48223/pf0000182167

Llanos, M. (2018). La cultura organizacional, principal propulsor de la calidad en Instituciones de Educación Superior: caso universidad ecuatoriana. Revista Espacios, 39(23), 29-44. Recuperado de https://www. revistaespacios.com/a18v39n23/a18v39n23p29.pdf

Licandro, O. D. \& Yepes, S. L. (2018). La Educación Superior conceptualizada como bien común: el desafío propuesto por Unesco. Revista Digital de 
Investigación en Docencia Universitaria, 12(1), 6-33. https://doi.org/10.19083/ridu.12.715

Martí-Noguera, J. J., Calderón A. I., \& Fernández-Godenzi, A. (2018). La responsabilidad social universitaria en Iberoamérica: Análisis de las legislaciones de Brasil, España y Perú. Revista Iberoamericana de Educación Superior, 9(24), 107-124. https://doi. org/10.22201/iisue.20072872e.2018.24.264

Martí-Noguera, J.J. \& Gaete, R. (2019). Construcción de un sistema de educación superior socialmente responsable en América Latina: Avances y desafíos. Revista Archivos Analíticos de Políticas Educativas, 27(97), 1-29. https://doi.org/10.14507/epaa.27.3925

Martí-Noguera, J.J., Licandro, O., \& Gaete-Quezada, R. (2018). La responsabilidad social de la Educación Superior como Bien Común. Concepto y Desafíos. Revista de la Educación Superior, 47(186), 1-22. https://doi. org/10.36857/resu.2018.186.353

Martí-Noguera, J.J. \& Martí-Vilar, M. (2013). Una década de responsabilidad social universitaria en Iberoamérica. Revista Española del Tercer Sector, 25, 145-161. Recuperado de http://roderic.uv.es/handle/10550/33669

Martí-Noguera, J.J., Martí-Vilar, M., \& Almerich, G. (2014). Responsabilidad social universitaria: influencia de valores y empatía en la auto atribución de comportamientos socialmente responsables. Revista Latinoamericana de Psicología, 46(3), 160-168. https:// doi.org/10.1016/S0120-0534(14)70019-6

Martí-Vilar, M. \& Rodríguez-Osorio, E. (2016). Formación en ética profesional y responsabilidad social universitaria aplicada a la psicología del envejecimiento saludable. En Arango, O. E. et al (Comp.). Ética profesional y responsabilidad social universitaria: universidad, sociedad y sujeto. (86-95). Medellín: FUNLAM

Martínez-Usarralde, M., Lloret-Catalá, C., \& Mas-Gil, S. (2017). Responsabilidad social universitaria (RSU): Principios para una Universidad Sostenible, Cooperativa y Democrática desde el Diagnóstico Participativo de su Alumnado. Revista Archivos Analíticos de Políticas Educativas, 25(75), 1-25. https://doi. org/10.14507/epaa.25.2769

Melewar, T., Karaosmanoglu, E., \& Paterson, D. (2005). Corporate identity: concept, components and contribution. Journal of General Management, 31(1), 5981. https://doi.org/10.1177/030630700503100104

Mendoza-Fernández, D., Salas-Solano, E., \& López Juvinao, D. (2015). Responsabilidad social en universidades públicas autónomas: Esencia académica con visión transformadora. Revista Clío América, 9(18), 135 -
142. https://doi.org/10.21676/23897848.1531

Ministerio de Educación (2019). Mi Futuro. Santiago de Chile. Recuperado de https://www.mifuturo.cl/buscador-de-instituciones/

Ministerio de Educación (2018). Ley N²1.094 sobre universidades estatales. Recuperado de https://www.leychile.cl/Navegar?idNorma $=1119253$

Montañez-García, A. (2017). Liderazgo, cultura organizacional y cambio en la universidad. Cuaderno de Inves tigación en la Educación, 32, 51-62. Recuperado de https://revistas.upr.edu/index.php/educacion/article/view/13923

Morales, F., Hollander, R., \& Valenzuela, N. (2017). Calidad de la información publicada en Internet en universidades chilenas. Capic Review, 15, 45-59. Recuperado de http://www.capic.cl/wp-content/uploads/2018/12/ VB-ARTICULO-4.pdf https://doi.org/10.35928/cr. vol15.2017.11

Navarro, G., Boero, P., Jiménez, G., Tapia, L., Hollander, R., Escobar, A., Baeza, M., \& Espina, Á. (2010). Universitarios y responsabilidad social. Revista Calidad en la Educación, 33, 101-121. http://dx.doi.org/10.31619/ caledu.n33.140

Navarro, G., Boero, P., Jiménez, G., Tapia, L., Hollander, R., Escobar, A., Baeza, M., \& Espina, Á. (2012). Valores y actitudes socialmente responsables en universitarios chilenos. Revista Calidad en la Educación, 36, 123-147. https://doi.org/10.4067/S0718-45652012000100004

Núñez, J.(2017).Losmétodosmixtosenlainvestigacióneneducación: Hacia un uso reflexivo. Cadernos de Pesquisa, 47(164), 632-649. Recuperado de http://www.scielo.br/pdf/cp/v47n164/1980-5314-cp-47-164-00632. pdf https://doi.org/10.1590/198053143763

Núñez, M., Alonso, I., \& Pontones, C. (2015). Responsabilidad social universitaria: estudio empírico sobre la fiabilidad de un conjunto de indicadores de Gobierno Corporativo. Revista Innovar, 25(58), 91-104. https:// doi.org/10.15446/innovar.v25n58.52428

Pérez, J. (2017). Universidad transparente: Transparencia activa focalizada para el ámbito universitario chileno. Revista Transparencia \& Sociedad, 5, 71-90. Recuperado de https://www.consejotransparencia.cl/ wp-content/uploads/2018/04/perez.pdf

Pérez-Mato, D., \& Borrazás-González, R. (2012). Aproximación a la concepción de valores compartidos en la dirección estratégica de una institución universitaria. VARONA Revista Científico-Metodológica, 55, 12-17. Recuperado de https://www.redalyc.org/articulo.oa?id=360633907003 
Piñuel, J. (2002). Epistemología, metodología y técnicas del análisis de contenido. Revista Estudios de Sociolingüística, 3(1), 1-42. Recuperado de http://web.jet.es/ pinuel.raigada/A.Contenido.pdf

Rodríguez-Ponce, E. \& Pedraja-Rejas, L. (2013). Dirección estratégica y calidad de las universidades: un estudio exploratorio desde Chile. Revista Interciencia, 38(1), 35-41. Recuperado de https://www.interciencia.net/ wp-content/uploads/2017/12/035-RODRIGUEZ-7.pdf

Rubio, C., Améstica-Rivas, L., King-Domínguez, A., \& Ganga-Contreras, F. (2019). Valor económico y atributos de la marca en universidades chilenas. Una aproximación a través del proceso de análisis jerárquico. Revista Interciencia, 44(8), 475-482. Recuperado de https://www.interciencia.net/wp-content/ uploads/2019/10/06_475_Com_Ganga_v44n8.pdf

Ruíz-Corbella, M., \& Bautista-Cerro, M. (2016). La responsabilidad social en la universidad española. Teoría de la Educación. Revista Interuniversitaria, 28(1), 159188. https://doi.org/10.14201/teoredu2016281159188

Solari, E. (2016). Antítesis chilenas sobre universidad, libertad y pluralismo. Revista Estudios Públicos, 143, 35 76. Recuperado de https://www.cepchile.cl/cep/site/ docs/20161004/20161004123553/rev143_esolari.pdf

Solís, F. \& Garduño, A. (2017). La responsabilidad social en la planeación estratégica institucional de las universidades públicas en México. Revista Internacional Administración \& Finanzas (IBFR), 10(4), 41-60. Recuperado de https://papers.ssrn.com/sol3/papers. cfm?abstract_id=3040336

Takaki, M., Bravo, R., \& Martínez, E. (2015). La gestión de la identidad corporativa en la Universidad: análisis y consecuencias desde la perspectiva del profesorado. Revista Europea de Dirección y Economía de la Empresa, 24, 25-34. https://doi.org/10.1016/j.redee.2014.05.001

Toniut, H., del Valle, S., Dondero, M., Molina, D., Nivollet,
D., Ordoñez, V., Pacheco, X., Quintana, A., \& Sordo, J. (2015). La gestión por valores: una mirada crítica sobre la gestión de organizaciones. Revista Argentina de Investigación en Negocios, 1(2), 57-69. Recuperado de http://redi.ufasta.edu.ar:8080/xmlui/ bitstream/handle/123456789/1570/7395-37796-3 PB.pdf?sequence=1

Valarezo, K. \& Tuñez, J. (2014). Responsabilidad social universitaria. Apuntes para un modelo de RSU. Revista de Comunicación, 13, 84-117. Recuperado de https://minerva.usc.es/xmlui/bitstream/handle/10347/18764/ Valarezo_ResponsabilidadSocialUniversitariaApuntesParaUnMod.pdf?sequence=1\&isAllowed=y

Vallaeys, F. (2008). Responsabilidad social universitaria: una nueva filosofía de gestión ética e inteligente para las universidades. Revista Educación Superior y Sociedad, 13(2), 193-220. Recuperado de http://unesdoc. unesco.org/images/0018/001820/182067s.pdf

Vallaeys, F. (2014). La responsabilidad social universitaria: un nuevo modelo universitario contra la mercantilización. Revista Iberoamericana de Educación Supe rior (RIES), 12(5), 105-117. https://doi.org/10.22201/ iisue.20072872e.2014.12.112

Vallaeys, F. (2018). Las diez falacias de la responsabilidad social universitaria. Revista Digital de Investiga ción en Docencia Universitaria, 12(1), 34-58. https:// doi.org/10.19083/ridu.12.716

Vallaeys, F. \& Álvarez, J. (2019). Hacia una definición latinoamericana de responsabilidad social universitaria. Aproximación a las preferencias conceptuales de los universitarios. Revista Educación XX1, 22(1), 93 116. https://doi.org/10.5944/educxx1.19442

Vidal-Martínez, I. (2019). Diálogo y gobierno multistakeholder en la Universidad. Sobre ética y responsabilidad social. Revista de Bioética y Derecho, 45, 179-196. Recuperado de http://scielo.isciii.es/pdf/ bioetica/n45/1886-5887-bioetica-45-00179.pdf

RIDU / Revista Digital de Investigación en Docencia Universitaria / e-ISSN: 2223-2516

(C) Los autores. Este artículo es publicado por la Revista Digital de Investigación en Docencia Universitaria del Área de Institutional Research and Effectiveness de la Dirección de Aseguramiento de la Calidad, Universidad Peruana de Ciencias Aplicadas. Este es un artículo de acceso abierto, distribuido bajo los términos de la LicenciaCreativeCommons Atribución-CompartirIgual 4.0 Internacional.(http://creativecommons.org/licenses/by-sa/4.0/), que permite el uso no comercial, distribución y reproducción en cualquier medio, siempre que la obra original sea debidamente citada. 